\title{
NOUVELle
}

\section{Rôle de l'hepcidine dans les infections cutanées}

Mariangela Malerba, Carole Peyssonnaux
${ }^{1}$ Université de Paris, Institut Cochin, Inserm U1016, CNRS UMR8104, 24 rue du Faubourg Saint Jacques, F-75014 Paris, France.

${ }^{2}$ Laboratoire d'excellence GR-Ex, Paris, France carole.peyssonnaux@inserm.fr
L’hepcidine hépatique, hormone clé de l'homéostasie du fer dans l'organisme L'hepcidine est un peptide de 25 acides aminés principalement produit par le foie et sécrété dans la circulation sanguine en conditions de surcharge en fer, d'infection, ou d'inflammation [1]. L'hepcidine est l'hormone de l'homéostasie du fer. Elle inhibe l'exportation du fer des entérocytes (site de l'absorption intestinale du fer alimentaire) et des macrophages (site de recyclage du fer de l'hémoglobine) en dégradant la ferroportine (FPN), une protéine exportatrice du fer située à la membrane de ces cellules [2]. Une augmentation de la production d'hepcidine provoque donc une diminution de la concentration plasmatique du fer. La carence en hepcidine est associée à l'hémochromatose héréditaire, une maladie caractérisée par une surcharge progressive en fer pouvant entraîner de graves dysfonctionnements de divers organes.

Le fer est le seul micronutriment dont la régulation hormonale dépend à la fois de l'état nutritionnel et d'une possible infection. Le fer joue un rôle particulier dans la médiation des interactions hôtepathogène, car ce métal est au centre d'une bataille nutritionnelle entre l'hôte et les pathogènes. L'hepcidine contribue à l'immunité innée en diminuant les niveaux de fer plasmatique, et ainsi la disponibilité du fer pour les microbes [3]. À terme, cependant, cette diminution du fer plasmatique associée à une inflammation conduit à une anémie.

L'hepcidine est également produite dans d'autres organes/cellules que le foie (rein, poumon, macrophages, etc.), notamment lors d'infections [4]. L'hepcidine produite par le foie étant suffisante pour assurer l'homéostasie systémique du fer dans des conditions physiologiques [5], la production d'hepcidine par les autres tissus pourrait jouer un rôle local, notamment au site des infections ou dans des tissus mal perfusés, inaccessibles à l'hepcidine présente dans le sang.

\section{Nouveau rôle immunomodulateur de I’hepcidine cutanée en conditions infectieuses}

Si l'hepcidine est désormais reconnue comme l'hormone de l'homéostasie du fer, elle a été identifiée initialement comme un peptide antimicrobien cationique (PAM) du fait de sa similarité de structure avec les $\beta$-défensines. Les PAM sont des antibiotiques naturels capables de tuer des souches bactériennes variées [6]. Même si l'hepcidine conserve les fonctions antimicrobiennes prototypiques des PAM dans différentes espèces de poissons, cette propriété ne persiste qu'à l'état rudimentaire chez les vertébrés supérieurs et n'a pas retenu l'attention de la communauté scientifique. Toutefois, les effets des PAM sont pléiotropes et assurent la protection de l'hôte non seulement par la destruction directe des agents pathogènes (fonction antimicrobienne) mais également par la modulation du système immunitaire de l'hôte (fonction immunomodulatrice). Pourtant, le rôle immunomodulateur de l'hepcidine et son éventuelle expression dans des épithéliums tels que celui de la peau (source majeure de production de PAM) n'avaient jamais été étudiés. Nous avons donc entrepris cette étude, en choisissant comme modèle la fasciite nécrosante (FN), plus connue sous le nom de gangrène ou «maladie mangeuse de chair».
La FN est une infection caractérisée par une nécrose généralisée de la peau et des tissus profonds, décrite pour la première fois par Hippocrate au Ve siècle. Le traitement standard comprend des antibiotiques à large spectre et un débridement chirurgical ${ }^{1}$ complet. Cependant, malgré l'usage d'antibiotiques et l'exérèse chirurgicale des tissus nécrotiques, le risque de septicémie reste élevé, avec un taux de mortalité pouvant atteindre $30 \%$. Le streptocoque du groupe A est considéré comme la cause la plus fréquente de FN associée à la septicémie [7].

Nous avons d'abord montré que I'hepcidine est produite par la peau de patients souffrant de FN due au streptocoque du groupe A [8]. Pour étudier son rôle dans le développement de la FN, nous avons utilisé un modèle murin d'infection nécrosante. Des bactéries d'une souche de streptocoque du groupe $A$ isolées à partir d'un patient atteint de FN sont introduites chez la souris par voie sous-cutanée; la souris développe alors des lésions nécrotiques de la peau et une septicémie dans les jours suivant l'infection. Comme chez I'homme, l'hepcidine est produite dans la peau infectée et peut être détectée dans les kératinocytes de l'épiderme. Grâce à l'analyse de souris mutantes dont le gène de l'hepcidine a été invalidé dans les kératinocytes, nous avons pu montrer que la production locale d'hepcidine est nécessaire non seulement pour limiter la prolifération bactérienne au site de la lésion mais également pour empêcher la dissémination bactérienne [8]. Nous avons alors cherché à comprendre le

\footnotetext{
1 Le débridement consiste à enlever tous les tissus dévitalisés, nécrotiques ou infectés.
} 


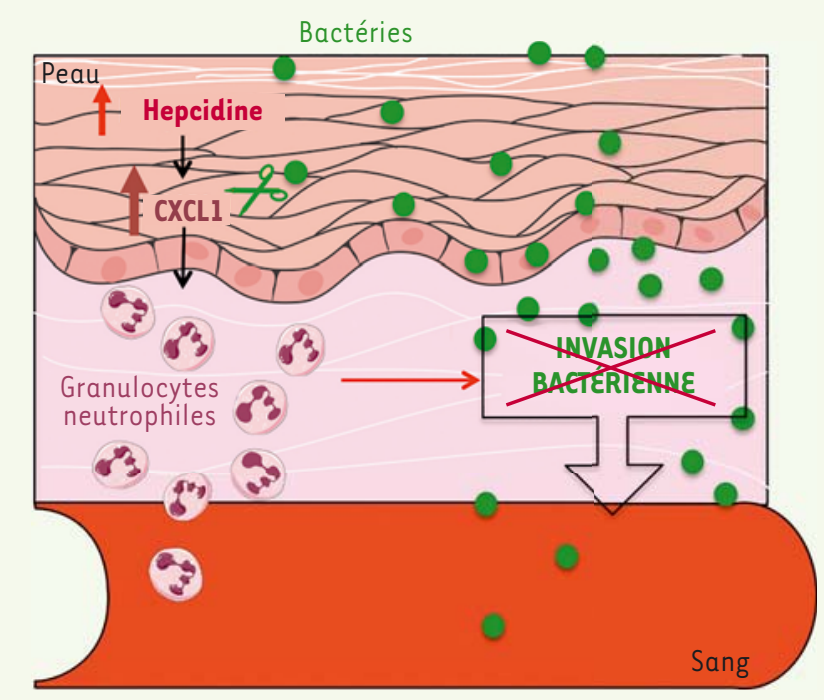

Figure 1. L'hepcidine produite par la peau augmente le recrutement des granulocytes neutrophiles au site d'infection et empêche l'invasion bactérienne. En réponse à l'infection par les streptocoques du groupe $A(S G A)$, les kératinocytes produisent l'hepcidine nécessaire à la synthèse de CXCLl, chimiokine clé des granulocytes (ou polynucléaires) neutrophiles. Contrairement à CXCLI, l'hepcidine est résistante à la dégradation par les principales protéases bactériennes (ici représentées par des ciseaux), et pourrait donc servir de réservoir pour maintenir des niveaux stables de CXCLI dans les tissus infectés. L'hepcidine permet alors un recrutement efficace de granulocytes neutrophiles, et empêche la propagation systémique des bactéries.

mécanisme en cause. L'hepcidine n'a pas d'effet antimicrobien direct sur ces bactéries. En revanche, l'hepcidine produite par les kératinocytes est essentielle à la synthèse de chemokine ( $C-X-C$ motif) ligand I (CXCL1), chimiokine clé des granulocytes neutrophiles. Cette induction semble s'effectuer par une voie dépendant de la ferroportine, seul récepteur connu de l'hepcidine. Cette augmentation de CXCL1 par l'hepcidine permet le recrutement des granulocytes neutrophiles, cellules clés du système immunitaire, au site de l'infection (Figure 1). Ces granulocytes sont des agents antibactériens essentiels dans la défense de l'organisme, notamment contre les infections par les streptocoques du groupe A. Lors de la détection de ces bactéries pyogènes, le recrutement des granulocytes neutrophiles, qui débute dès les premières minutes de l'infection, est également essentiel à sa résolution [9]. Toutefois, les streptocoques du groupe $\mathrm{A}$ contrecarrent le système immunitaire de
I'hôte en produisant des protéases, telles que SpyCEP, qui vont dégrader CXCLl et d'autres protéines impliquées dans le recrutement et l'activation des granulocytes neutrophiles [10]. Nous avons montré que l'hepcidine, contrairement à CXCLl, est résistante à la dégradation, ce qui suggère qu'elle pourrait servir de réservoir pour maintenir un pool de CXCLI dans le contexte de l'infection. CXCLI est phylogénétiquement ancien puisqu'il est déjà présent chez l'amibe Dictyostelium discoideum, tandis que l'hepcidine est apparue plus récemment au cours de l'évolution, chez les poissons téléostéens. Compte tenu de la théorie selon laquelle les interactions hôte-pathogène co-évoluent, nous pouvons spéculer que les streptocoques du groupe A ont évolué pour contrer l'activité de CXCLI, alors qu'ils n'ont pas encore développé de facteurs de virulence capables de neutraliser l'activité de l'hepcidine.

Nous avons fait l'hypothèse que l'hepcidine, du fait de sa résistance à la dégra- dation et de ses propriétés immunomodulatrices, pourrait être utilisée dans un but thérapeutique. En effet, l'administration de l'hepcidine à des souris dans les 24 heures suivant l'infection par des streptocoques du groupe A stimule le recrutement de granulocytes neutrophiles et empêche l'invasion bactérienne [8]. L'utilisation thérapeutique de l'hepcidine peut donc constituer une stratégie alternative opportune compte tenu de l'incidence croissante des maladies invasives dues aux streptocoques du groupe $A$.

$\varepsilon$ conclusion de cette étude, nous avons montré que l'hepcidine, outre son rôle clé en tant qu'hormone de régulation du fer produite par le foie, est également un composant essentiel, jusqu'ici non reconnu, de la réponse immunitaire à une infection bactérienne lorsqu'elle est produite par l'épiderme. L'hepcidine possède des rôles pléiotropes en conditions infectieuses. Elle restreint la disponibilité du fer pour les microorganismes, favorise le recrutement des granulocytes neutrophiles, et empêche la dissémination bactérienne. Elle est très résistante aux protéases bactériennes, contrairement aux chimiokines des granulocytes neutrophiles. Comme l'hepcidine n'agit pas sur les bactéries elles-mêmes, mais sur le système immunitaire et sur la disponibilité du fer, un nutriment clé pour les bactéries, nous pouvons supposer que son action thérapeutique ne se limite pas aux streptocoques du groupe $A$, mais s'applique à un large éventail de bactéries. L'hepcidine pourrait donc être envisagée comme une alternative thérapeutique aux antibiotiques dans de nombreuses maladies. $\diamond$

Role of hepcidin in cutaneous infections

\section{LIENS D'INTÉRÊT}

Les auteures déclarent n'avoir aucun lien d'intérêt concernant les données publiées dans cet article.

\section{RÉFÉRENCES}

1. Nicolas G, Chauvet C, Viatte L, et al. The gene encoding the iron regulatory peptide hepcidin is regulated by anemia, hypoxia, and inflammation. J Clin Invest $2002 ; 110$ : 1037-44. 


\section{RÉFÉRENCES}

2. Ganz T. Systemic iron homeostasis. Physiol Rev 2013 ; $93: 1721-41$.

3. Weiss G, Ganz T, Goodnough LT. Anemia of inflammation. Blood $2019 ; 133: 40-50$.

4. Daher R, Lefebvre T, Puy H, Karim Z. Extrahepatic hepcidin production: the intriguing outcomes of recent years. World J Clin Cases 2019 ; 7 : 1926-36.
5. Zumerle S, Mathieu JR, Delga S, et al. Targeted disruption of hepcidin in the liver recapitulates the hemochromatotic phenotype. Blood 2014 ; 123 : 3646-50.

6. Nakatsuji T, Gallo RL. Antimicrobial peptides: old molecules with new ideas. J Invest Dermatol 2012 ; $132: 887-95$.

7. Hakkarainen TW, Kopari NM, Pham TN, Evans HL. Necrotizing soft tissue infections: review and current concepts in treatment, systems of care, and outcomes. Curr Probl Surg $2014 ; 51$ : 344-62.
8. Malerba M, Louis S, Cuvellier S, et al. Epidermal hepcidin is required for neutrophil response to bacterial infection. J Clin Invest 2020 ; 130 : 329-34.

9. Walker MJ, Barnett TC, McArthur JD, et al. Disease manifestations and pathogenic mechanisms of group A Streptococcus. Clin Microbiol Rev 2014 ; 27 : 264301.

10. Dohrmann S, Cole JN, Nizet V. Conquering neutrophils. PLoS Pathog 2016 ; 12 : el005682.

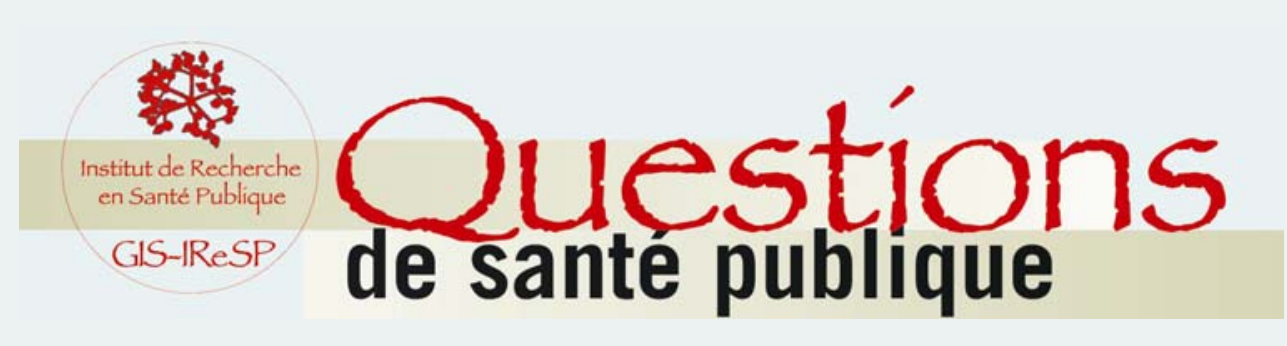

\title{
Un nouveau bulletin
}

\author{
pour une meilleure \\ visibilité des résultats \\ de la recherche \\ en santé publique
}

es résultats de la recherche en santé publique souffrent en France d'un réel manque de visibilité. Ceci concerne aussi bien le monde académique (hors santé publique) que le grand public et les décideurs. Pour pallier ce déficit, I'IReSP a créé un bulletin à large diffusion intitulé "Questions de santé publique ", largement inspiré du bulletin mensuel d'information de I'INED «Populations et sociétés ". L'objectif éditorial est de porter à la connaissance d'un large public (enseignants, étudiants, journalistes, décideurs, milieux de la recherche, associations, public concerné) les informations les plus récentes concernant des questions importantes de santé publique, rédigées de façon facilement lisible et compréhensible pour des non spécialistes, en garantissant que les informations publiées sont validées scientifiquement. La publication concerne des faits et non des positions. Audelà de la présentation de résultats, les qualités pédagogiques de Questions de santé publique permettent au lecteur de mieux comprendre comment sont formulées et abordées les questions de santé publique et quelles sont les limites de ces études.

Nom

Prénom

Institution

Fonction

Spécialité

Service

Adresse

Ville

Code postal

Pays

Adresse électronique

à nous retourner par la poste ou par fax au 0149850345

Questions de santé publique

EDP Sciences

17 avenue du Hoggar

91944 Les Ulis

France
Réservé aux abonnés de M/S

Recevez gratuitement et régulièrement

Questions de santé publique

en renvoyant ce document soigneusement rempli.

Questions de santé publique est une publication de l'Institut de Recherche en Santé Publique. I Directeur de Ia publication : Corinne Alberti. I Rédactrice en chef : Kodja Yetongnon. I Comité de relecture : Lorraine Cousin, Jean-Marie Gagliolo, Coline Terroba. I Réalisation : EDP Sciences. 